\title{
Study on the sensitivities and damage mechanisms of ultra-low permeability sandstone reservoirs: taking Chang 6 reservoir in Jingbian oilfield as an example
}

\author{
Xiulan Zhu ${ }^{1, *}$, Yanlong Ran $^{2}$, Zhanjun $\mathrm{Chen}^{1}$, Tai Xu ${ }^{1}$, Shengling Jiang ${ }^{1}$, and Guangsheng Zhang ${ }^{1}$ \\ ${ }^{1}$ Longdong University, College of Energy Engineering, Qingyang745000, China \\ ${ }^{2}$ Gansu Energy Group Company Limited, Qingyang745000, China
}

\begin{abstract}
This paper takes the ultra-low permeability sandstone reservoir of Jingbian oilfield in Ordos Basin as the research object, analyzes the petrological characteristics, diagenesis, physical characteristics and pore structure characteristics of the reservoir, and carries out reservoir sensitivity evaluation by using rock casting thin sections, $\mathrm{X}$-ray diffraction, and sensitive flow experiments. The research results show that the ultra-low permeability Chang 6 sandstone reservoir has weak velocity sensitivity, medium-weak water sensitivity, weak salt sensitivity, weak alkali sensitivity and strong acid sensitivity; the damage mechanism of reservoir sensitivity mainly depends on the composition of clay minerals and pore structure after diagenesis. The clay mineral content from high to low is chlorite, illite, a small amount of illite / smectite layer, and kaolinite, of which the chlorite content is as high as $75 \%$; the reservoir has poor physical properties, the types of small hole-thin throat and small hole-fine throat. The reservoir is prone to blockage such as bridge plugging. Therefore, ultra-low permeability sandstone reservoirs are prone to different degrees of sensitivity. The reservoir characteristics are consistent with the reservoir sensitivity evaluation results.
\end{abstract}

\section{Introduction}

At present, the proportion of proven oil and gas reserves of low-permeability oil fields in my country is increasing year by year, and has great development potential [1-2]. The Jingbian oilfield in the Ordos Basin is a typical lowpermeability oilfield. This type of oilfield has the characteristics of "low permeability, low production, and low pressure". Measures such as acid fracturing and water injection development are usually considered during the development process to ensure high and stable production. Due to the high content of clay minerals in ultra-low permeability sandstone reservoirs (especially the high content of sensitive minerals), the reservoir space is mostly composed of micropores and fine throats, with poor seepage capability, which leads to more complex reservoir sensitivity [1-2]. Therefore, it is necessary to conduct research on reservoir protection and formation damage evaluation, analyze the interaction mechanism of reservoir-sensitive minerals and external working fluids, reservoir seepage capacity, and evaluate reservoir sensitivity [3-5] to achieve the purpose of efficiently developing ultra-low permeability sandstone oil fields.

Taking Chang 6 reservoir of Jingbian oilfield in Ordos Basin as the research object, this paper evaluates its sensitivity characteristics and analyzes the basic characteristics of reservoirs comprehensively to study the mechanism of reservoir sensitivity damage by the laboratory experiments, which provides a certain reference for similar reservoirs.

\section{Basic characteristics of the reservoir}

\subsection{Petrological characteristics}

According to the test results analysis of the casting thin section experiment, X-ray diffraction experiment, scanning electron microscope experiment, the Chang 6 reservoir rock are mainly light gray and dark gray medium-fine and fine-grained feldspar sandstone. Among them, the clastic component is mainly feldspar, with a content of $41 \sim 70 \%$; the secondary component is quartz, with a content of $22 \sim 35 \%$, and the content varies in different lithologies. The content of quartz in siltstone can reach $40 \%$. $\sim 45 \%$; the content of cuttings is generally $5 \sim 15 \%$ (Figure 1), and it also contains a small amount of mica, mainly biotite.

The content of cement in the Chang 6 reservoir rock is relatively high, with an absolute content of $10 \sim 16 \%$, and its composition is complex (Figure 1), mostly carbonate, clay, laumontite, etc. The main type of cementation is porous cementation. The reservoir contains carbonate cement, which is mainly composed of calcite and part of which is iron calcite; its content in 
sandstone varies widely, ranging from 1 to $30 \%$. when its content is low, it fills the pores in the form of stars; when its content is high, the sand layer forms a flaky contiguous cementation, which makes the sandstone denser, forming a dense interlayer in the sand layer [6]. Clay mineral cements in the reservoir rocks are mainly chlorite and are produced in the form of thin films, which have a positive effect on the preservation of primary pores and the formation of secondary pores. It also contains a small amount of illite and illite / Smectite layers, the ratio between illite / Smectite layers is mostly $<10$.

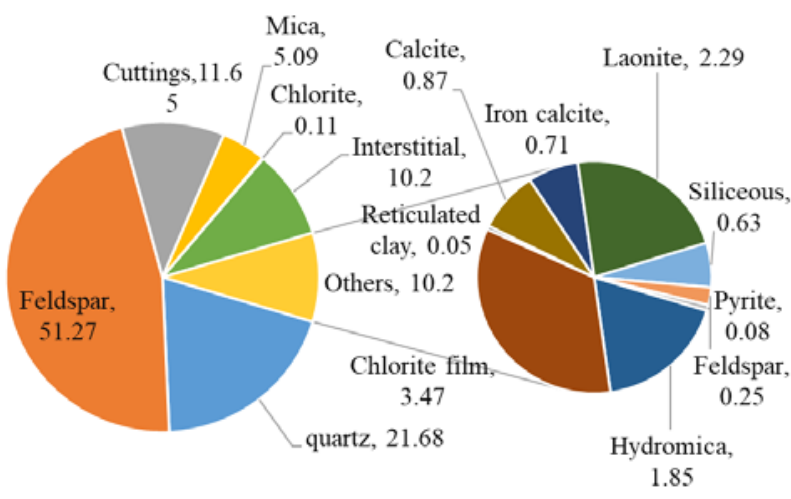

Fig.1. Schematic diagram of mineral composition in reservoir rocks.

\subsection{Diagenesis}

The diagenesis of the Chang 6 reservoir is relatively strong, having undergone diagenetic epigenesis such as compaction, dissolution, cementation recrystallization, and authigenic mineral filling and evolved into a sandstone reservoir with low porosity and low permeability. Debris particles in this reservoir have medium roundness, basically sub-edge-sub-circular, medium sorting, and the contact mode between reservoir particles is mainly point-line, local bump contact and a few suture contact. Therefore, the Chang 6 reservoir has the typical characteristics of low sandstone mineral maturity and high structural maturity.

\subsection{Characteristics of pore structure}

The pore size of Chang 6 reservoir is mainly distributed in the range of $5 \sim 100 \mu \mathrm{m}$, and the overall distribution is nearly normal distribution, mainly $20 \sim 50 \mu \mathrm{m}$, accounting for $55.14 \%$ of the total sample. The pore types of the reservoir are mainly intergranular pores, dissolution pores and a small amount of intercrystalline pores and micro-cracks. The intergranular pores are mainly the primary intergranular pores and the remaining intergranular pores. The remaining intergranular pores are the majority. Most of the pore walls and pores are filled with clay minerals. The filling is mainly chlorite, a small amount of illite and illite / Smectite layers. The dissolution pores are mainly feldspar pores and laumontite pores, and the degree of dissolution is uneven, which is controlled by sedimentation and diagenesis. The type of reservoir throats are the small hole-thin throat and the small holefine throat. The pore-throat sorting performance is relatively poor. The average sorting coefficient is 2.331 .

\subsection{Physical characteristics}

Based on the statistical analysis of the physical properties of natural cores in Chang 6 reservoir, the effective porosity of Chang 6 reservoir is $4.6 \% \sim 14.8 \%$, the average porosity is $10.38 \%$; the permeability is $0.097 \sim 198 \times 10^{-3} \mu^{2}$, and the average permeability is $1.378 \times 10^{-3} \mu \mathrm{m}^{2}$, the correlation between porosity and permeability is small, that is, porosity has no obvious control effect on permeability. It is a typical lowporosity and ultra-low permeability sandstone reservoir.

\section{Evaluation of reservoir sensitivity}

Although the sedimentary compaction of ultra-low permeability sandstone reservoirs is obvious, the pore structure of the reservoir is still very complicated. Therefore, the ultra-low permeability sandstone reservoir sensitivity flow experiment requires higher measurement accuracy of the device and the lower flow rate during the test. The indoor experiments strictly follow "SY/T53582010 Reservoir Sensitivity Flow Experimental Evaluation Method". The rock samples used in the experiment are all natural cores of Chang 6 reservoir, and the fluid used is simulated formation water or oil field water.

\subsection{Evaluation of velocity sensitivity}

The velocity sensitivity experiment must be preceded by other sensitivity experiments to prevent velocity sensitivity from affecting the results of other sensitivity evaluation experiments. The purpose of the experiment is to determine the reasonable injection-production rate of each well. In the velocity sensitivity experiment, different injection rates (from small to large) are used to inject formation water into the core, and the permeability of the rock is measured at different injection rates. In the experiment, the flow rate of $0.1,0.25,0.5,0.75,1.0,1.5$ and $2.0 \mathrm{ml} / \mathrm{min}$ was used to test and calculate the permeability damage rate $D_{v}$ of the reservoir velocity sensitivity in sequence, and the calculation formula is as follows:

$$
\begin{gathered}
D_{\mathrm{v}}=\frac{\left|K_{\mathrm{n}}-K_{\mathrm{i}}\right|}{K_{\mathrm{i}}} \times 100 \% \\
D_{v}=\max \left(D_{v 2}, D_{v 3}, \ldots, \quad D_{v n}\right)
\end{gathered}
$$

In the formula:

$D_{v n}$ - permeability change rate of the rock sample at different flow rate, $\%$;

$K_{n}$ - permeability of the rock sample at different flow rates, $\times 10^{-3} \mu \mathrm{m}^{2}$;

$K_{i}$-initial permeability of the rock sample, $\times 10^{-3}$ $\mu \mathrm{m}^{2}$;

$D_{v}$ - velocity sensitivity damage rate of rock sample, $\%$. 
The velocity sensitivity evaluation experiment results show that the maximum permeability damage rate of the rock sample is $14.0 \%$, the critical fluid velocity is 0.75 $\mathrm{ml} / \mathrm{min}$, and the velocity sensitivity of the reservoir is generally weak. High water injection rate will damage the formation, so it is better to consider that the injection-production rate of Chang 6 reservoir should not exceed $0.75 \mathrm{ml} / \mathrm{min}$.

\subsection{Evaluation of Water sensitivity}

The purpose of the reservoir water sensitivity evaluation experiment is to analyze the generation mechanism of the hydration expansion, dispersion and migration of clay minerals after encountering fresh water, and to determine various working fluid formulations. The simulated water used in the experiment was oil field water with a salinity of $5 \times 10^{4} \mathrm{ppm}$. Formation water, sub-formation water and distilled water were used to inject the rock samples in sequence, and their measured permeability are $0.753 \times 10^{-3} \mu \mathrm{m}^{2}, 0.529 \times 10^{-3} \mu \mathrm{m}^{2}$, $0.504 \times 10^{-3} \mu^{2}$, respectively. The test calculation results show that the maximum damage rate of the rock sample is $33.07 \%$, which is moderate to weak water sensitivity. It can be seen that formation water is the best injection water.

\subsection{Evaluation of salt sensitivity}

The purpose of the reservoir salt sensitivity evaluation experiment is to evaluate the degree of permeability decline due to the change of the salinity of the injected fluid, and to find the critical salinity that leads to a significant drop in permeability. In the experiment, fluids with a salinity of $6.0,5.5,5.0,4.5,4.0$ and $3.5 \times$ $10^{4} \mathrm{ppm}$ (salinity from high to low) were injected into the rock sample in sequence, and the permeability of the rock sample under different salinities was measured and calculated.

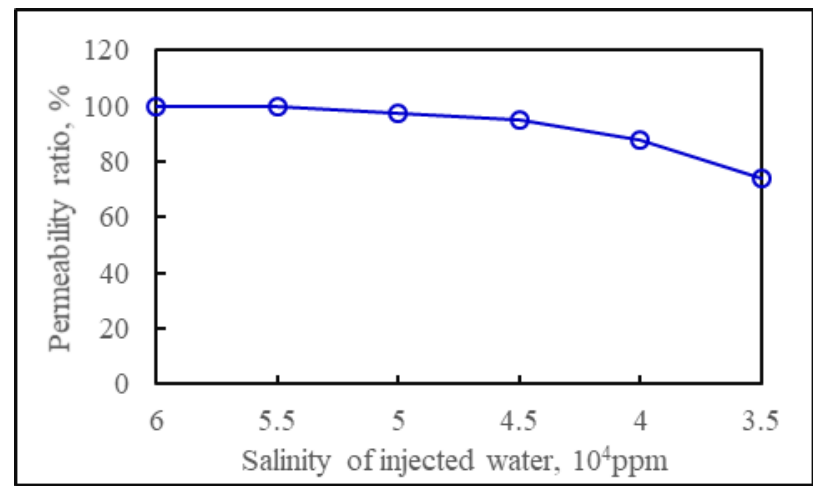

Fig.2. Salt sensitivity experiment curve.

According to the experimental test results (Figure 2), it can be seen that the critical salinity must not be lower than $4.0 \times 10^{4} \mathrm{ppm}$. Low-salinity water is not suitable for injecting into the formation. If the salt content is $3.5 \times$ $10^{4} \mathrm{ppm}$, the permeability damage rate of the rock sample is $26.24 \%$, which is weakly salt sensitive and will not have a significant impact on the later water injection development.

\subsection{Evaluation of acid sensitivity}

Due to the small pores and fine throats and poor fluid seepage capacity of ultra-low permeability sandstone reservoirs, hydraulic fracturing or acidification must be used to improve the pore structure and connectivity of the reservoir and increase the oil production of oil wells. The acid sensitivity evaluation experiment was directed to injecting $13 \% \mathrm{HCL}+3 \% \mathrm{HF}$ into the rock sample. The permeability of the rock sample before and after the injection of the mixed acid solution is measured and calculated to be $18.93 \times 10^{-3} \mu^{2}, 11.31 \times 10^{-3} \mu \mathrm{m}^{2}$. The sample permeability damage rate is $40.25 \%$, which is a strong acid sensitivity. Therefore, the Chang 6 sandstone reservoir is not suitable for various acid treatment measures.

\subsection{Evaluation of alkali sensitivity}

The $\mathrm{pH}$ of the formation water is usually neutral or slightly alkaline. Most of the external working fluid has a high $\mathrm{pH}$ value. Once it is injected into the reservoir, there will be incompatibility between the injected working fluid and the reservoir, resulting in the destruction of the structure of clay minerals and siliceous cements in the reservoir, particles released after the dissociation of clay minerals and dissolution of cements, which eventually causes reservoir blockage and reduced permeability. The purpose of the alkali sensitivity evaluation experiment is to analyze the change process of reservoir permeability due to the change of $\mathrm{pH}$ value of external working fluid $(\mathrm{pH}=7,8,9,10,11)$, and to evaluate the degree of reservoir damage caused by alkali sensitivity. It is convenient to determine the $\mathrm{pH}$ value of the external working fluid.

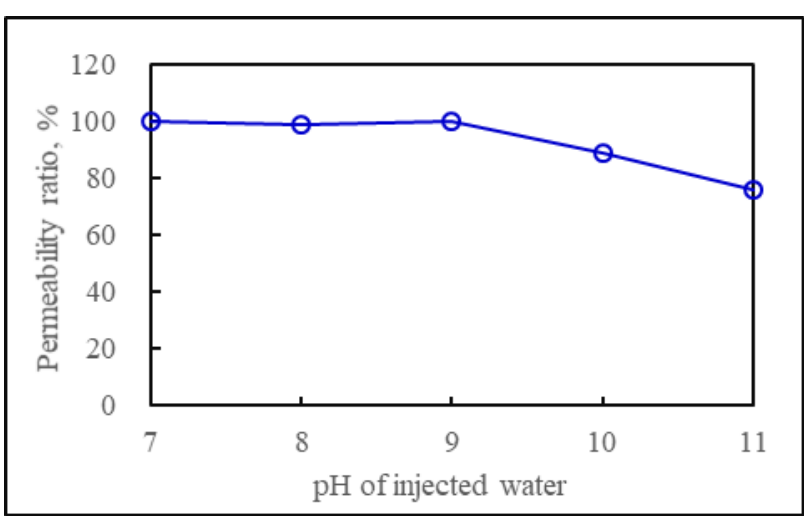

Fig.3. Alkaline sensitivity test curve.

The alkali sensitivity test results show (Figure 3) that the critical $\mathrm{pH}$ value of the injected fluid is 9 , and the alkalinity of the injected fluid should not be too strong. When the fluid $\mathrm{pH}$ value is equal to 11 , the permeability damage rate of the rock sample determined by the experiment is $23.69 \%$. The degree of the alkali sensitive damage is weak alkali sensitive. 


\section{Mechanism of sensitive damage}

In oilfield development, the difference in the nature and flow rate of the injected external working fluid will cause hydration expansion, physical and chemical adsorption, cementation precipitation, unstable particle migration and accumulation of reservoir clay minerals [7-9]. Reservoir pores become smaller, throats are blocked, and permeability drops drastically, resulting in poorer reservoir performance and oilfield development effects.

The composition and content of clay minerals in the rock mineral composition of the reservoir [10] are the key factors for reservoir sensitivity. Kaolinite has cleavage, low hardness, small interlayer force, and weak resistance to mechanical damage. Under the impact of high-speed external working fluid, it is prone to cleavage and cracking, particle migration and velocity sensitivity. Smectite and illite are prone to cation replacement. It causes water molecules to enter from between the crystal layers, montmorillonite and illite are hydrated, resulting in water sensitivity and salt sensitivity. When the ironrich chlorite encounters acid, it will react to form acidsensitive mineral precipitation to produce an acidsensitive. Quartz particles, clay minerals and other aluminosilicate minerals in the reservoir undergo a dissolution reaction under strong alkali conditions [4]. The migration and accumulation of solid particles and colloids or gels produced by dissolution can easily block pore throats and cause layer damage to produce alkali sensitivity.

Clay minerals in the Chang 6 reservoir rock samples accounted for $4.95 \%$ of the total rock composition and $53.36 \%$ of the interstitials. Clay minerals are mainly chlorite, accounting for about $75 \%$ of the total, followed by illite, with a small amount of illite / smectite layer and kaolinite with uneven distribution. According to the experimental analysis of rock casting thin sections, leafshaped chlorites and a small amount of ribbon-shaped illite are distributed between and on the surface of rock samples. Chlorite membranes are mostly distributed in leaf-shaped perpendicular to the surface of the clastic particles. In the medium-fine-grained feldspar sandstone and lithic feldspar sandstone with chlorite membranes, the clastic particles are mostly in point-line contact, the strength of mechanical compaction is weak, and the residual intergranular pores are developed; In sandstones with underdeveloped stone membranes and high argillaceous base content, mechanical compaction is strong. The common quartz and feldspar are enlarged. The acid-sensitive mineral chlorite of Chang 6 reservoir has a large distribution range, so the acid sensitivity of Chang 6 reservoir is relatively strong, but the velocity sensitivity, water sensitivity, salt sensitivity and alkali sensitivity of Chang 6 reservoir are weak.

\section{Conclusion}

(1) The Chang 6 reservoir in Jingbian Oilfield is dominated by light gray and dark gray medium-fine and fine-grained feldspar sandstone. The pores are dominated by intergranular pores and dissolved pores. The throats are the type of small hole-thin throat and small hole-fine throat; clay minerals accounted for $4.95 \%$ of the total rock composition, mainly chlorite accounting for about $75 \%$ of the total clay minerals, followed by illite, a small amount of illite/smectite layers and kaolinite. As a result, Chang 6 reservoirs have varying degrees of sensitivity.

(2) A sensitivity evaluation experiment was carried out on the Chang 6 reservoir rock samples in Jingbian oilfield. The sensitivity of Chang 6 reservoir is "five weak and one strong", weak velocity sensitivity, medium-weak water sensitivity, weak alkali sensitivity, weak salt sensitivity and strong acid sensitivity.

(3) Reservoir sensitivity analysis is an important condition for evaluating the adaptability of reservoirs to different development methods and measures. In the oilfield development process, the compatibility of the injected working fluid with the reservoir should be considered to achieve the purpose of reservoir protection.

\section{Acknowledgements}

This research was financially supported by Gansu Provincial Industrial Green Low-Carbon Transformation and Upgrading Project (Project Nos. GGLD-2019-060), 2020 Gansu Provincial Association for Science and Technology Innovation Driven Engineering Demonstration Project (Project Nos. ZLGC202003-4), Longdong University Youth Science and Innovation Fund Project (Project Nos. XYZK1906) and Longdong University Education and Teaching Research Project (Project Nos. 2019-42).

\section{References}

1. Lu J L, Wu Y Y, Petroleum Geol Oilfield Dev Daqing, 38(2019)

2. Leng $X$ G, Zhang $Z$ G, Chen J W et al, Petroleum Geol Oilfield Dev Daqing, 35(2016)

3. Zhan D, Xu H, Wang L et al, Lith Reserv, 28(2016)

4. Shao D B, Chen J W, J Xi'an Shiyou Univ (Nat Sci Ed), 32(2017)

5. Chai G S, Shi Y M, Du S H et al, Acta Sci Naturalium Universitatis Pekinensis (Nat Sci Ed), 56(2020)

6. Dang B, Zhao H, Kang X Y et al, J Cent S Univ (Sci Technol), 44(2013)

7. Wang $\mathrm{Y} X$, Zhou L F, Jiao Z S et al, J Jilin Univ (Earth Sci Ed), 48(2018)

8. He Y H, Fault-Block Oil Gas Field, 21(2014)

9. Kang X, Hu W X, Wang J et al, J Chin Univ Min Technol, 46(2017)

10. Peng $\mathrm{S} \mathrm{M}$, Yin $\mathrm{X}$, Zhang $\mathrm{J} \mathrm{C}$ et al, Acta Petrolei Sinica, 27(2006) 